\title{
Imaging techniques in treatment algorithms of pulmonary embolism
}

\author{
M. Pistolesi*, M. Miniati
}

Imaging techniques in treatment algorithms of pulmonary embolism. M. Pistolesi, M. Miniati. (C) ERS Journals Ltd 2002.

ABSTRACT: Pulmonary embolism (PE) is more often diagnosed post mortem by pathologists than in vivo by clinicians. The identification of practical diagnostic algorithms could reduce the rate of diagnoses first made at autopsy.

The literature was reviewed for evidence-based approaches to PE diagnosis. Since the PE mortality rate greatly exceeds that of deep vein thrombosis (DVT), more emphasis was given to reports specifically dealing with $\mathrm{PE}$ diagnosis by objective pulmonary vascular imaging techniques than to those aimed at DVT detection.

Several studies have shown that standardized clinical estimates can be effectively used to give a pretest probability to calculate, after appropriate objective testing, the post-test probability of PE. A prospective trial has shown that perfusion scanning, rather than ventilation/perfusion scanning, should be the imaging technique of first choice for the management of patients suspected of having PE. The clinical usefulness of spiral computed tomography has not as yet been firmly established. However, ongoing technological developments would probably render the technique accurate enough to replace conventional angiography.

The authors propose a noninvasive diagnostic algorithm with high predictive accuracy (positive predictive value 96\%; negative predictive value $98 \%$ ) starting with a standardized assessment of clinical likelihood, followed by a perfusion scan and, eventually, spiral computed tomography in only a minority of patients $(<20 \%)$ with discordant clinical and scintigraphic findings.

Eur Respir J 2002; 19: Suppl. 35, 28s-39s.

\begin{abstract}
*Respiratory Medicine Unit, Dept of Critical Care, University of Florence, Florence and "Pulmonary Unit, National Research Council, Institute of Clinical Physiology, Pisa, Italy.
\end{abstract}

Correspondence: M. Pistolesi, Dept of Critical Care, Respiratory Medicine Unit, University of Florence, Viale Morgagni, 85, 50134 Firenze, Italy. Fax: 390554223202

E-mail: massimo@unifi.it

\section{Keywords: Chest computerized}

tomography

chest radiography

echocardiography

lower limb ultrasonography

lung scanning

pulmonary embolism

Received: August 102001

Accepted August 312001
A general consensus regarding the diagnostic procedures for pulmonary embolism (PE) has not as yet been developed. Different specialists emphasize different approaches to the diagnosis of this very common and life-threatening disease, and, therefore, different and often contrasting opinions are continuously reported in the extensive international literature on the subject. This is a peculiarity of PE, which, after acute myocardial infarction and stroke, is the third commonest acute cardiovascular emergency. Myocardial infarction and stroke are diagnosed according to widely accepted guidelines. In explaining this discrepancy, it should be borne in mind that, on the one hand, PE has a more elusive clinical presentation and, on the other, most hospitals do not have clinical units dedicated to the diagnosis of PE. The diagnosis of PE is thus more dependent on the opinions and disparate competencies of the specialist who is occasionally confronted with this clinical problem than on standardized and evidence-based approaches likely to confirm or exclude the disease with high predictive accuracy.

These considerations may help explain why PE is more often diagnosed post mortem by pathologists than in vivo by clinicians [1-4]. It has been shown that $\sim 70 \%$ of the major (either fatal or contributing to death) pulmonary embolic events diagnosed at autopsy have been overlooked by the attending clinicians [5]. Standardization of the diagnostic work-up with the multidisciplinary integration of different competencies is the only means of reducing the number of diagnoses first made at autopsy.

No diagnostic work-up would even start without awareness of the high incidence of the disease in the general population [5-7]. Accuracy of clinical evaluation (based on the presence of predisposing risk factors, symptoms, signs and a few readily available instrumental examinations) is the first fundamental and mandatory step in raising clinical suspicion of the disease and setting-up diagnostic strategies. The number of clinically undetected major episodes of PE can only be significantly reduced by means of diagnostic approaches aimed at diagnosing the disease across its entire broad spectrum of clinical presentation. Minimizing the percentage of patients in whom the disease is revealed only once the syndrome of haemodynamic instability and hypotension has already developed can prevent death from PE. In other words, both the overall number of diagnoses and the percentage of patients who are diagnosed at an early stage must increase.

In the present article, a diagnostic algorithm based on standardized clinical evaluation combined with objective pulmonary vascular imaging tests, whose 
predictive accuracy has been prospectively assessed by comparison with pulmonary angiography, is presented. The general principles of the algorithm, which are more fully described in the appropriate sections, are as follows: 1) each patient clinically suspected of having PE should undergo accurate clinical evaluation to establish a clinical likelihood of the disease prior to further objective testing; and 2) each patient should undergo imaging of the pulmonary circulation for definitive confirmation or exclusion of the disease and, with a few noticeable exceptions, the diagnostic work-up of the patients should not be terminated after a positive lower limb ultrasound examination, a negative D-dimer test or a negative echocardiogram. These general principles rely on the tenet that pretest clinical probability is the cornerstone of any objective and effective diagnostic process [8], the accuracy of a diagnostic technique can be firmly established only by comparing it with a standard reference technique rather than by performing outcome clinical studies [9], and the risk of early death after diagnosis is much higher in patients with $\mathrm{PE}$ compared to those with isolated deep vein thrombosis (DVT) [10].

\section{Pretest clinical probability of pulmonary embolism}

\section{Clinical suspicion of pulmonary embolism}

The clinical diagnosis of PE is thought to be unreliable because symptoms, signs and laboratory data are often nonspecific [11, 12]. However, it has long been recognized that clinical findings such as unexplained dyspnoea or chest pain, either or both of which are observed in $97 \%$ of patients with proven $P E$, may be useful in raising suspicion of $P E$ and selecting patients for further diagnostic testing [13-16]. However, the aforementioned symptoms are not specific to PE inasmuch as they are encountered, with nearly equal frequency, in a variety of acute cardiopulmonary disorders including myocardial infarction, lung oedema, aortic dissection, pneumonia and pneumothorax. Therefore, it is necessary to complement the information obtained by clinical history taking and physical examination with additional data derived from a few readily available laboratory tests such as chest radiography, electrocardiography and arterial blood gas analysis. Interpretation of these additional data may either strengthen the clinical suspicion of PE or suggest alternative diagnoses. For example, radiographic evidence of pulmonary oedema or pneumothorax makes the diagnosis of PE less likely. Conversely, enlargement of the descending pulmonary artery with signs of abrupt vascular cut-off [17], focal areas of reduced density with loss of vascular markings (focal oligaemia) [18] and parenchymal areas of pleurally based consolidation suggestive of infarction [19] are radiographic findings compatible with PE. Likewise, electrocardiographic signs of acute right ventricle strain $[20,21]$ and arterial hypoxaemia with respiratory alkalosis [22] may contribute, in the appropriate clinical setting, to reinforce the suspicion of PE.
Although the process of integrating clinical and instrumental data is somewhat empirical, it is surprisingly accurate. The results of two broad prospective studies on the diagnosis of PE [23, 24] indicate that: 1) physicians' estimates of the clinical likelihood of PE do have predictive value; and 2) clinical likelihood can be used as a pretest probability to calculate the post-test probability of PE after appropriate objective testing. Furthermore, three recent articles [25-27] have shown that well-characterized clinical estimates of pretest probability of PE can be used for the safe management of patients suspected of having the disease. The results of the PISA-PED trial [26], because it is the only study in which the performance of the clinical diagnostic algorithm was objectively and prospectively evaluated using pulmonary angiography as reference diagnostic standard, are summarized here. The prevalence of the clinical features observed in the Prospective Investigative Study of Acute Pulmonary Embolism Diagnosis (PISA-PED) [26] is compared with that obtained in the Prospective Investigation of Pulmonary Embolism Diagnosis (PIOPED) study [23], the only other large-scale trial in which the diagnosis or exclusion of PE was based on the results of pulmonary angiography.

\section{Clinical history}

On interviewing patients with suspected PE, it is important to elicit a detailed clinical history in order to identify predisposing risk factors and pertinent clinical symptoms. The most relevant findings in a population of 500 consecutive patients with clinical suspicion of PE are reported in table 1 [26].

Risk factors. Prolonged immobilization, recent surgery or trauma, a history of thrombophlebitis and cancer are considered to be associated with a significantly higher risk of PE [28]. In the PISAPED study [26], at least one of these risk factors was present in $>80 \%$ of patients with established PE and in nearly $70 \%$ of those without. The high prevalence of risk factors in patients in whom PE was subsequently excluded does not permit the use of this information for assessing with certainty the clinical likelihood of PE. These results are at variance with those obtained in two other prospective studies in which the assignment of a clinical probability was weighted on the presence or absence of risk factors $[25,27]$.

Symptoms. In the PIOPED study [14-16], unexplained dyspnoea and pleuritic chest pain were by far the most frequent symptoms reported by patients with confirmed PE, and tachypnoea and tachycardia the most frequent clinical signs. However, the prevalence of these symptoms and signs was not significantly different from that observed in patients who turned out not to have PE [14-16]. In the PISAPED study [26], sudden-onset dyspnoea, chest pain (either pleuritic or substernal) and fainting were present, either singly or in combination, in $96 \%$ of patients with a PE and in $59 \%$ of those without. Because of their high sensitivity, 
the absence of these symptoms had a $94 \%$ negative predictive value for PE. However, due to their low specificity $(41 \%)$, the presence of these symptoms in various combinations had a positive predictive value of only 53\% [26]. Thus, by relying on the presence or absence of these symptoms alone, a correct diagnosis or exclusion of PE would have been made in no more than $60 \%$ of patients [26].

Physical signs. As can be seen from table 1, signs revealed on physical examination were not of great

Table 1.-Risk factors, symptoms, physical signs and routine instrumental findings in 500 consecutive patients suspected of pulmonary embolism (PE)

\begin{tabular}{|c|c|c|c|}
\hline & $\begin{array}{c}\text { PE } \\
\text { present }\end{array}$ & $\begin{array}{c}\text { PE } \\
\text { absent }\end{array}$ & p-value \\
\hline Subjects $n$ & 202 & 298 & \\
\hline \multicolumn{4}{|l|}{ Risk factors } \\
\hline Immobilization $>3$ days $*$ & $119(59)$ & $138(46)$ & 0.007 \\
\hline Surgery* & $81(40)$ & $116(39)$ & 0.72 \\
\hline Thrombophlebitis (ever) & $69(34)$ & 57 (19) & 0.0002 \\
\hline Cardiovascular diseases & $53(26)$ & $96(32)$ & 0.18 \\
\hline Bone fractures (lower limb)* & $46(23)$ & $36(12)$ & 0.002 \\
\hline Neoplastic diseases $\#$ & $36(18)$ & 43 (14) & 0.37 \\
\hline \multicolumn{4}{|l|}{ Symptoms } \\
\hline \multicolumn{4}{|l|}{ Dyspnoea } \\
\hline Sudden onset & $158(78)$ & $87(29)$ & $<0.0001$ \\
\hline Gradual onset & $12(6)$ & $59(20)$ & 0.0002 \\
\hline \multicolumn{4}{|l|}{ Chest pain } \\
\hline Pleuritic & $89(44)$ & $89(30)$ & 0.002 \\
\hline Substernal & $33(16)$ & $29(10)$ & 0.04 \\
\hline Fainting & $53(26)$ & $38(13)$ & 0.0002 \\
\hline Haemoptysis & 19 (9) & $16(5)$ & 0.12 \\
\hline Cough & $22(11)$ & $45(15)$ & 0.22 \\
\hline Palpitation & 36 (18) & $46(15)$ & 0.56 \\
\hline \multicolumn{4}{|l|}{ Signs } \\
\hline Tachycardia $>100$ beats $\cdot \min ^{-1}$ & $48(24)$ & $69(23)$ & 0.96 \\
\hline Cyanosis & 33 (16) & $44(15)$ & 0.73 \\
\hline Neck vein distension & $25(12)$ & $28(9)$ & 0.009 \\
\hline Leg swelling (unilateral) & $35(17)$ & $27(9)$ & 0.009 \\
\hline Crackles & 37 (18) & $76(26)$ & 0.08 \\
\hline \multicolumn{4}{|l|}{ Routine instrumental findings } \\
\hline \multicolumn{4}{|l|}{ Electrocardiography } \\
\hline $\begin{array}{l}\text { Signs of right ventricular } \\
\text { overload }\end{array}$ & $100(50)$ & $35(12)$ & $<0.00001$ \\
\hline \multicolumn{4}{|l|}{ Chest radiography } \\
\hline Right heart enlargement & $77(38)$ & $43(14)$ & $<0.00001$ \\
\hline Amputation of hilar artery & $72(36)$ & $3(1)$ & $<0.00001$ \\
\hline Oligaemia & $91(45)$ & $3(1)$ & $<0.00001$ \\
\hline $\begin{array}{l}\text { Pleurally based } \\
\text { consolidation }\end{array}$ & $31(15)$ & $2(1)$ & $<0.00001$ \\
\hline Platelike atelectasis & $65(32)$ & $70(23)$ & 0.04 \\
\hline $\begin{array}{l}\text { Unilateral elevated } \\
\text { diaphragm }\end{array}$ & $86(43)$ & $89(30)$ & 0.005 \\
\hline $\begin{array}{l}\text { Bilateral elevated } \\
\text { diaphragm }\end{array}$ & $39(19)$ & $57(19)$ & 0.95 \\
\hline Pleural effusion & $91(45)$ & $104(35)$ & 0.02 \\
\hline \multicolumn{4}{|l|}{ Gas tensions } \\
\hline$P \mathrm{a}, \mathrm{O}_{2} \mathrm{mmHg}$ & $65 \pm 13$ & $68 \pm 18$ & 0.02 \\
\hline$P \mathrm{a}, \mathrm{CO}_{2} \mathrm{mmHg}$ & $32 \pm 4$ & $34 \pm 5$ & 0.0001 \\
\hline
\end{tabular}

*: within 4 weeks of study entry; ${ }^{*}$ : clinically active malignancy with pathological diagnosis within 3 months of study entry. Data are presented as $\mathrm{n}(\%)$ or mean $\pm \mathrm{SD} . \mathrm{Pa}, \mathrm{O}_{2}$ : arterial oxygen tension; $P$ a, $\mathrm{CO}_{2}$ : arterial carbon dioxide tension. $1 \mathrm{mmHg}=0.133 \mathrm{kPa}$. help in differentiating patients with proven PE from those without. The only two physical signs prevailing in patients with confirmed PE were neck vein distension and unilateral leg swelling (table 1). However, these findings had too low a prevalence to be of value among patients suspected of having PE in differentiating those affected from the disease from those unaffected. Further information, which can be derived from simple and readily available laboratory tests such as electrocardiography, chest radiography and measurement of arterial blood gas levels, is, therefore, required.

\section{Instrumental examination}

Electrocardiography. Electrocardiographic results were abnormal in $70 \%$ of patients with PE with no prior cardiopulmonary disease who were enrolled in the PIOPED study [15]. Nonspecific changes in the ST segment and T-wave were the most frequent abnormalities, occurring in some $50 \%$ of patients with established PE [15]. In the PISAPED study [26], electrocardiographic signs of acute right ventricular overload were found in $50 \%$ of patients with proven PE (table 1). Electrocardiographic abnormalities suggestive of right ventricular overload included $\mathrm{T}$-wave inversion in the right precordial leads $(23 \%)$, S1Q3/ S1Q3T3 (19\%), transient right bundle branch block $(9 \%)$, pseudoinfarction $(6 \%)$ and S1S2S3 (3\%). These abnormalities turned out to be fairly specific for PE because they were observed in only $12 \%$ of patients in whom the disease had been excluded [26].

Chest radiography. Contrary to widespread belief, a normal chest radiograph is seldom observed in patients with PE. In the PIOPED study, only $12 \%$ of the chest radiographs from 383 patients with $\mathrm{PE}$ were rated as normal [29]. The most frequent radiographic abnormalities were atelectasis and pulmonary parenchymal consolidation, either or both of which occurred in $69 \%$ of patients with $\mathrm{PE}$ and $58 \%$ of those without [29]. Oligaemia, prominent central pulmonary artery, pleurally based opacities and an elevated diaphragm were found to be poor predictors of the disease. It was concluded, therefore, that the main value of chest radiography is in excluding conditions that may mimic the clinical presentation of PE [29]. In the PISAPED study [26], only $14 \%$ of patients with PE had normal chest radiography results. Oligaemia, amputation of the hilar artery and pulmonary consolidations compatible with infarction were present in 45,36 and $15 \%$ of patients, respectively (table 1). Even though the sensitivity of each of these radiographic abnormalities was low, at least one of them was present in nearly $75 \%$ of patients with proven PE. By way of contrast, these three radiographic abnormalities were observed in only $1 \%$ of patients without PE. Thus, it appears that the chest radiograph is useful in not only excluding PE but also, due to the high specificity of some findings, strengthening the suspicion of the disease when interpreted in conjunction with other clinical information [26]. 
Arterial blood gas abnormalities. Arterial hypoxaemia with respiratory alkalosis is a common feature of acute PE. In the PIOPED study [15], the arterial oxygen tension $\left(\mathrm{Pa}, \mathrm{O}_{2}\right)$ while breathing room air was $<10.6 \mathrm{kPa}$ in $81 \%$ of patients with PE. Similarly, in the PISAPED study, as many as $90 \%$ of patients with $\mathrm{PE}$ had a $\mathrm{Pa}, \mathrm{O}_{2}$ of $<10.9 \mathrm{kPa}$ and an arterial carbon dioxide tension $\left(\mathrm{Pa}_{\mathrm{a}} \mathrm{CO}_{2}\right)$ of $<4.9 \mathrm{kPa}$ [26]. It should be recognized, however, that significant arterial hypoxaemia and hypocapnia may occur in clinical conditions other than PE. In the PISAPED study, 75\% of patients in whom $\mathrm{PE}$ was excluded had a $\mathrm{Pa}, \mathrm{O}_{2}$ of $<10.0 \mathrm{kPa}$ and a $P \mathrm{a}, \mathrm{CO}_{2}$ of $<4.9 \mathrm{kPa}$ [26]. Thus, physicians should not be led astray by the finding of arterial hypoxaemia with respiratory alkalosis if other important elements supporting the diagnosis of PE are absent, or if clinical, electrocardiographic and radiographic findings suggest an alternative diagnosis. These results have been confirmed by a recent survey showing that arterial blood gas levels are not predictive in confirming or excluding the diagnosis in patients suspected of having PE [30]. Arterial blood gas abnormalities remain, however, of great clinical value in the process of raising the suspicion of the disease and in the determination of gas exchange impairment in patients with established PE, during both the acute phase and follow-up after therapy.

\section{Diagnostic algorithm for assessing the pretest clinical probability of pulmonary embolism}

The present authors have developed a clinical diagnostic algorithm that includes the identification of three symptoms (sudden-onset dyspnoea, chest pain and fainting) with high sensitivity and negative predictive value and their association with electrocardiographic (acute right ventricular overload) and radiographic (oligaemia, amputation of hilar artery and consolidations compatible with infarction) abnormalities with high specificity and positive predictive value [26]. These symptoms (singly or in combination) were associated with at least one of the aforementioned electrocardiographic and radiographic abnormalities in 164 of $202(81 \%)$ patients with PE, and in only 22 of $298(7 \%)$ patients without [26]. In a validation group of 250 consecutive patients with clinical suspicion of the disease, the sensitivity and specificity of the clinical diagnostic algorithm were 84 and 95\%, respectively [26].
Although the diagnostic algorithm turned out to be fairly accurate, it failed to identify $\sim 20 \%$ of patients with PE on angiography. In those patients in whom the algorithm was falsely negative, the severity of the disease, in terms of the unperfused vascular bed, was significantly less than in those who were correctly classified as having PE. Furthermore, whenever the three symptoms included in the diagnostic algorithm were not associated with the electrocardiographic or radiographic abnormalities described previously or were associated only with electrocardiographic signs of acute right ventricular overload, the positive predictive value for PE was not $>50 \%$ [26]. It appears, therefore, that PE cannot be diagnosed or excluded with certainty by clinical assessment alone. However, on the basis of the diagnostic algorithm, it was possible to categorize the pretest clinical probability of the disease as low, intermediate or high (table 2) [26]. In 583 consecutive patients with an abnormal lung scan in whom pulmonary angiography was used as reference diagnostic standard, PE was present in as many as 227 of $234(97 \%)$ patients with a high clinical probability, 67 of $145(46 \%)$ with an intermediate clinical probability and only 12 of $204(6 \%)$ with a low clinical probability [26]. Because of their high predictive accuracy, the standardized clinical estimates of PE reported in the PISA-PED trial can be used as a pretest probability to calculate, after further objective testing, the post-test probability of PE.

In the PIOPED study [23], experienced physicians rated the clinical probability of PE as low $(0-19 \%)$, intermediate $(20-79 \%)$ or high $(80-100 \%)$. No standardized clinical diagnostic algorithm was used. PE was diagnosed in $68 \%$ of patients with a high clinical probability, and in only $9 \%$ of those who were assigned a low clinical probability [22]. In those patients who had been rated as having an intermediate clinical probability (64\% of the entire cohort), the prevalence of PE was 30\% [23].

In a Canadian multicentre prospective study, the clinical probability of PE was categorized as low, intermediate or high according to a standardized clinical model which included assessment of presenting symptoms and signs, risk factors for venous thromboembolism, and presence or absence of an alternative diagnosis at least as likely as PE [25]. The prevalence of $\mathrm{PE}$ in the low, intermediate and high pretest probability categories was 3,28 and $78 \%$, respectively.

More recently, WICKI et al. [27] proposed a

Table 2. - Standardized criteria for establishing a pretest clinical probability of pulmonary embolism

\begin{tabular}{ll}
\hline Probability & Criteria \\
\hline High & $\begin{array}{c}\text { Presence of at least one of three symptoms (sudden-onset dyspnoea, chest pain and fainting) } \\
\text { not otherwise explained and associated with at least one of three radiographic findings } \\
\text { (amputation of the hilar artery, oligaemia and pleurally based consolidation) } \\
\text { Presence of at least one of three symptoms (sudden-onset dysnoea, chest pain and fainting) } \\
\text { not otherwise explained but not associated with the aforementioned electrocardiographic } \\
\text { and radiographic abnormalities, or associated only with electrocardiographic signs of } \\
\text { acute right ventricular overload } \\
\text { Absence of the aforementioned symptoms or identification of an alternative diagnosis that } \\
\text { may account for their presence }\end{array}$ \\
Low &
\end{tabular}


standardized probability score based on the evaluation of several variables: age, risk factors, hypocapnia, hypoxaemia, tachycardia and radiographic findings (band atelectasis and monolateral elevation of a hemidiaphragm). PE was diagnosed in $10 \%$ of patients classified as having a low probability, $38 \%$ of those with an intermediate probability and $81 \%$ of those with a high probability [27]. However, using this score, only $6 \%$ (63 of 986) of patients were classified as having a high probability, whereas low and intermediate probabilities were assigned to 49 and $44 \%$ of patients, respectively. Thus, the proposed score seems more appropriate for identifying those patients with a low clinical probability to be studied less aggressively than for use in the classification of the whole population of patients suspected of having PE.

\section{Pulmonary vascular imaging techniques}

Among the many diagnostic techniques that have found an application in patients with a clinical suspicion of PE, imaging of the pulmonary circulation still retains the most relevant role. The addition of spiral computed tomographic angiography [31] to the previously introduced lung scanning and conventional pulmonary angiography has opened a still ongoing debate among imaging specialists as to the relative role of this new application of computed tomography (CT) with respect to older techniques [32-36]. A specific role for magnetic resonance imaging of the chest in the diagnosis of PE has also been advocated [37]; however, the predictive accuracy of this technique has not as yet been prospectively evaluated in large-scale clinical trials. Lung scanning, spiral computed tomographic angiography and conventional pulmonary angiography (alone or in combination) are, at the present time, the most commonly employed pulmonary vascular imaging techniques for establishing the presence or absence of PE. Conventional pulmonary angiography is still the reference standard against which other diagnostic imaging and nonimaging diagnostic approaches should be validated before being introduced into clinical practice.

\section{Lung scanning}

The continuous search for new diagnostic strategies probably stems from the unsuccessful application to the diagnosis of $\mathrm{PE}$ of ventilation/perfusion lung scanning $\left(V^{\prime} / Q^{\prime}\right.$ scan). Ventilation was added to perfusion lung scanning ( $Q^{\prime}$ scan) with the aim of increasing the specificity of the procedure. This technique, which continues to be widely used, is based on the false premise that ventilation should be normal in lung regions with embolic vascular obstruction. This assumption is at variance with the pathophysiological notion that dead space ventilation is not invariably increased in patients with PE [38] and the observation that the increase in wasted ventilation in lung regions with embolic vascular obstruction, when present, is not as prominent as the reduction in pulmonary perfusion [22]. As the late J.H. Comroe Jr anticipated, in 1966, before the introduction of $V^{\prime} / Q^{\prime}$ scanning into clinical practice, "the decrease in wasted ventilation helps the patients but hinders the physician in the diagnosis" [38]. The definitive proof that the $V^{\prime} / Q^{\prime}$ scan is of limited diagnostic value comes from the results of the PIOPED trial, which showed that the high-probability $V^{\prime} / Q^{\prime}$ scan (i.e. perfusion defects with matching normal ventilation) fails to detect the disease in some $60 \%$ of patients with angiographically proven PE (sensitivity $41 \%$ and specificity 97\%) [23]. Further analysis of the PIOPED data has shown that in a large proportion of the lung regions with pulmonary vascular obstruction on angiography there is radiographic evidence of atelectasis or consolidation [29]. It is obvious that the ventilation scan cannot be normal in those lung regions. Hence, according to the recommendations of the PIOPED trial, definitive diagnosis of $\mathrm{PE}$ in most patients relies on pulmonary angiography [23].

The more recent PISA-PED trial [24] was aimed at assessing prospectively by comparison with pulmonary angiography the predictive accuracy of the $Q^{\prime}$ scan in patients suspected of PE. In this study, the ventilation scan was omitted and the $Q^{\prime}$ scan images were classified according to the shape of the perfusion defects, regardless of their number and size, and the presence of matching radiographic abnormalities. Table 3 reports the criteria used in the PISA-PED trial for $Q^{\prime}$ scan interpretation. At variance with the PIOPED study, in which the $V^{\prime} / Q^{\prime}$ scan is classified in terms of probability, the interpretation of the abnormal $Q^{\prime}$ scan in the PISA-PED study is dichotomous, either suggestive or not suggestive of PE. The sensitivity and specificity of the abnormal $Q^{\prime}$ scan compatible with PE, when corrected for the selection bias in referring patients for angiography, were 86 and $93 \%$, respectively [24].

According to these results, the $Q^{\prime}$ scan rather than the $V^{\prime} / Q^{\prime}$ scan can be considered the imaging technique of first choice after initial clinical evaluation in the development of diagnostic strategies likely to confirm or exclude the presence of PE. This opinion is based on the following considerations: 1) a normal or near-normal $Q^{\prime}$ scan excludes PE [39-41]; 2) an abnormal $Q^{\prime}$ scan can be interpreted dichotomously as suggestive or not suggestive of PE with high predictive accuracy [24]; 3) a two-fold increase in the sensitivity of the scintigraphic procedure was

Table 3.-Perfusion scan categories and interpretation criteria

\begin{tabular}{ll}
\hline Category & Criteria \\
\hline Normal & $\begin{array}{l}\text { No perfusion detects } \\
\text { Impressions caused by enlarged } \\
\text { heart, hila or mediastinum are } \\
\text { seen on an otherwise-normal scan } \\
\text { Abnormal, }\end{array}$ \\
$\begin{array}{l}\text { Single or multiple wedge-shaped } \\
\text { perfusion defects }\end{array}$ \\
$\begin{array}{c}\text { Abnormal, not } \\
\text { suggestive of PE }\end{array}$ & $\begin{array}{l}\text { Single or multiple perfusion defects } \\
\text { other than wedge-shaped }\end{array}$ \\
\hline
\end{tabular}

PE: pulmonary embolism. 
demonstrated when the lung scans of the PIOPED study were read using only the perfusion images without the ventilation scan [24]; 4) the $Q^{\prime}$ scan is the least expensive of the pulmonary imaging techniques [42], is easy to perform and has virtually no contraindications; 5) the perfusion defects revealed by the $Q^{\prime}$ scan could be a useful guide for angiographic techniques (either conventional or spiral CT), possibly increasing their sensitivity and specificity [26]; and 6) the $Q^{\prime}$ scan is the most practical means in the followup of patients with PE to evaluate the progression of the disease and the effect of anticoagulant treatment.

\section{Spiral computed tomographic angiography}

Unlike lung scanning (either $V^{\prime} / Q^{\prime}$ or $Q^{\prime}$ ), whose accuracy, assessed by comparison with pulmonary angiography in large-scale prospective trials [23, 24], will not change significantly in the future, the clinical usefulness of spiral computed tomographic angiography in the diagnostic work-up of PE has not as yet been firmly established. With the ongoing technological advancements leading to improvements in data acquisition, particularly the use of thinner section collimation and multidetector CT, both the accuracy and clinical utilization of spiral computed tomographic angiography are likely to increase.

Many reports since 1999 have shown that spiral computed tomographic angiography has such a high negative predictive value in ruling out a diagnosis of $\mathrm{PE}$ as to be proposed as an alternative to lung scanning and even to conventional pulmonary angiography [43-56]. Furthermore, spiral CT is, reportedly, able to provide additional ancillary information suggesting or confirming alternative diagnoses in patients with a clinical suspicion of PE. However, the positive results of these studies are mitigated by several considerations regarding the methodology applied to obtain the reported data. With only one exception [49], the results of spiral CT were not prospectively evaluated in comparison with pulmonary angiography; clinical outcome without anticoagulation, together with other nondefinitive tests for the objective assessment of PE status, was instead used in most studies as the reference standard. The accuracy of clinical follow-up in reliably assessing the value of a diagnostic test for PE has been questioned [9]. Moreover, assuming that patients with negative spiral CT results might be affected by more minor forms of the disease, the low recurrence and death rates from PE observed by withholding anticoagulant treatment for a short follow-up period cannot be taken as definitive proof of the high negative predictive value of the test. Patients with nonsevere forms of PE could have a favourable outcome even if left untreated [57].

Given the poor predictive accuracy of the $V^{\prime} / Q^{\prime}$ scan and the high rate of nondiagnostic results for subsequent objective clinical management, it is not surprising that spiral CT has been proved to be more useful than lung scanning in the diagnostic work-up of patients suspected of PE. However, none of the referred studies has made comparisons in terms of accuracy between the results obtained by spiral CT and those by $Q^{\prime}$ scan, either alone or in combination with the pretest clinical likelihood of PE. The proposal of omitting the lung scan altogether is questionable for at least three main reasons: 1) a normal $Q^{\prime}$ scan rules out with an accuracy greater than that of spiral CT clinically significant PE and renders unnecessary further diagnostic testing; 2) the perfusion defects on the $Q^{\prime}$ scan could possibly be used to guide a targeted computed tomographic reconstruction in those lung regions with the greatest abnormalities; and 3) once the diagnosis of PE has been established by whatever approach, it would be inappropriate to follow the progression of the disease using spiral computed tomographic angiography instead of the $Q^{\prime}$ scan.

Further limitations of spiral computed tomographic examination are the generally observed high rate ( $\sim 10 \%$ or even higher) of suboptimal or inconclusive results, the experience- and training-dependent interobserver variability and the observation that alternative diagnostic findings on CT are of limited value in differentiating between patients with PE and those without, since additional findings are almost equally present in both conditions [58].

Four meta-analyses on the role of spiral CT in the diagnosis of PE published in 2000 and 2001 clearly indicated the lack of prospective controlled studies and that the technique is not sensitive enough to detect subsegmental clots [59-62]. A prospective study in unselected consecutive outpatients has demonstrated too low a negative predictive value $(82 \%)$ to allow PE to be ruled out without further testing [63].

The reported sensitivity and specificity of spiral computed tomographic angiography range 53-100\% and $81-100 \%$, respectively [59]. It is difficult to obtain a meaningful assessment of mean sensitivity and specificity given the small size, and broad spectrum of the different clinical characteristics, of the published series of patients. However, considering the weighted means for the sensitivity and specificity of spiral CT reported in the meta-analysis by RATHBUN et al. [59] for patients with pulmonary angiography, mean values of 85 and $92 \%$, respectively, were computed by the present authors. These mean values for sensitivity and specificity were derived from the results of papers dealing with embolic events at the lobar and segmental and subsegmental vessel level. It is conceivable that these estimates are conservative figures as the reported meta-analysis [59] was based on studies performed 1992-1999 with instrumentation that is now becoming obsolete. Incessant technological advances in this technique will probably soon lead to increases in both sensitivity and specificity that will render spiral computed tomographic angiography accurate enough to replace conventional pulmonary angiography.

\section{Other instrumental evaluation}

Instrumental evaluation not allowing imaging of the pulmonary circulation is often used in the noninvasive diagnostic approach to patients with a 
clinical suspicion of PE. These tests are not specifically aimed at revealing embolic obstruction of the pulmonary vascular bed but at detecting: 1) circulating substances indicating the presence of venous thromboembolism (D-dimer); 2) the presence of DVT (lower limb compression ultrasonography); or 3) the acute right ventricular dysfunction resulting from PE (echocardiography). As such they have specific limitations in the definitive confirmation or exclusion of the pulmonary vascular embolic process.

\section{$D$-dimer test}

During the 1990s, there has been growing interest in the exploitation of newer laboratory tests in the noninvasive diagnostic work-up of patients suspected of having PE. Among them, measurement of the plasma concentration of D-dimer has been extensively investigated. D-dimer is a specific breakdown product released into the systemic circulation by endogenous fibrinolysis of cross-linked fibrin clots. Plasma D-dimer concentrations above a given cut-off level (usually $0.5 \mathrm{mg} \cdot \mathrm{L}^{-1}$ ) have a high sensitivity $(>95 \%$ ) but a low specificity $(<50 \%)$ for venous thromboembolism [64]. Because of the high sensitivity of the test, D-dimer concentrations of $<0.5 \mathrm{mg} \cdot \mathrm{L}^{-1}$ have a strong negative predictive value for venous thromboembolism ( $>95 \%$ in most studies). However, due to the poor specificity of the test, D-dimer concentrations of $>0.5 \mathrm{mg} \cdot \mathrm{L}^{-1}$ are not useful to confirm a diagnosis of venous thromboembolism (positive predictive value $<60 \%$ in most reports). Other clinical conditions such as myocardial infarction, stroke, sepsis, malignancy, liver disease, recent surgery or trauma may be associated with elevated plasma concentrations of D-dimer [64]. The reported sensitivity and specificity of the test are in relation to quantitative assays, such as the enzyme-linked immunosorbent assay (ELISA), lower sensitivity, precluding the utilization of the test as a screening method for the exclusion of venous thromboembolism without integrating it with clinical probability or further objective testing, has been found for more rapid and practical D-dimer determinations $[65,66]$. Furthermore, excessive prescription of the test and poor compliance of physicians in adhering to published guidelines reduce its costeffectiveness in the normal clinical setting [67].

\section{Lower limb compression ultrasonography}

This procedure has gained widespread acceptance as a noninvasive means for diagnosing DVT. From direct comparisons with contrast venography, ultrasonography shows $>97 \%$ sensitivity and specificity for proximal DVT in symptomatic patients, but is relatively insensitive in detecting isolated calf vein thrombosis [68] or proximal DVT in asymptomatic patients [69]. The test is somewhat complementary to the determination of D-dimer because it is clinically useful only when positive. Reportedly, DVT is diagnosed by noninvasive venous studies in $<50 \%$ of patients with proven PE [70]. Therefore, a single negative ultrasonography result does not permit, in itself, the exclusion of PE, especially if this entity is strongly suspected on clinical grounds. To overcome this limitation, serial leg testing over a 2-week period has been implemented with the aim of detecting eventual thrombotic extension from the distal to the proximal venous segment [25]. Whether such an approach is cost-effective remains to be firmly established. A recent retrospective study has shown that, in patients with a high clinical likelihood of $\mathrm{PE}$ and a low-probability $V^{\prime} / Q^{\prime}$ scan, it is not safe to terminate the diagnostic work-up after a negative lower extremity venous ultrasound examination [71]. Furthermore, another study, based on the prospective evaluation of the clinical utility of lower limb compression ultrasonography in patients with a nondiagnostic $V^{\prime} / Q^{\prime}$ scan, has shown that the posttest probability of PE after a single negative bilateral leg vein study is too high $(12 \%)$ to preclude therapeutic decisions without further diagnostic studies [72]. Conversely, a diagnostic strategy based on serial leg testing may be impractical in many clinical settings.

\section{Echocardiography}

The contribution of transthoracic echocardiography to the diagnosis of $\mathrm{PE}$ is based on the possible detection of emboli in the right heart cavities and central pulmonary arteries, and in its ability to reveal acute dysfunction of the right ventricle secondary to PE. Additionally, echocardiography could help to identify other cardiovascular conditions, such as acute myocardial infarction, dissection of the aorta and pericardial tamponade, which may clinically mimic PE. However, the direct visualization of emboli "in transit" within the right heart chambers or the proximal pulmonary circulation can be considered an almost exceptional event, and abnormalities of the right ventricle can be detected in clinical studies in only $\sim 50 \%$ of cases of PE [73, 74].

Only a few studies have addressed prospectively the diagnostic accuracy of echocardiography in patients with suspected PE [75-77]. These studies have yielded conflicting results with broad sensitivity (51-93\%) and specificity $(82-94 \%)$ ranges. This may result from differences in clinical setting, patient selection criteria, severity of PE and criteria adopted for confirming the disease.

A study aimed at determining prospectively by comparison with pulmonary angiography the sensitivity and specificity of thransthoracic echocardiography in unselected consecutive patients suspected of having PE gave a sensitivity and specificity of 56 and $90 \%$, respectively [78]. Echocardiographic findings of right ventricle dysfunction had a positive predictive value of $100 \%$ for PE when coupled with a high or intermediate pretest clinical probability. Conversely a negative echocardiogram associated with a low clinical pretest probability gave a negative predictive value of $98 \%$ [78]. When echocardiographic results and clinical probability were discordant, the post-test probability of PE was neither sufficiently 
high nor sufficiently low to confirm or exclude the disease with any certainty [78]. In the latter group of patients (a third of the cohort), the prevalence of PE was $51 \%$. Patients with true positive transthoracic echocardiography results had, on average, a significantly higher number of unperfused lung segments on $Q^{\prime}$ scan than those with a false negative examination (8.8 versus $5.7, \mathrm{p}<0.0001)$ [78].

It appears, therefore, that a negative echocardiographic study does not rule out PE, especially when the disease has a high clinical pretest probability. Detection of right ventricular dysfunction in patients with PE is, conversely, of great clinical value in assessing the severity of disease and long-term prognosis [74, 77, 79-81].

\section{Clinical probability combined with further objective testing}

Although the diagnostic yield of individual clinical symptoms and signs and common laboratory test results is limited, combination of these variables, by either empirical assessment [23, 24, 82] or a prediction rule [25-27], can be used to express a clinical probability of PE. The latter may serve as a pretest probability for predicting the probability of PE after further objective testing (post-test probability).

In the PIOPED study, a high clinical probability combined with a high probability $V^{\prime} / Q^{\prime}$ scan had a 96\% positive predictive value, whereas a low clinical probability paired with a normal/near-normal or lowprobability $V^{\prime} / Q^{\prime}$ scan had a $97 \%$ negative predictive value [23]. Unfortunately, some $75 \%$ of patients in the PIOPED study did not fit into these categories [23]. Other combinations of clinical and lung scan findings were of little diagnostic value [23].

The study protocol of WeLLs et al. [25] included a standardized clinical probability of $\mathrm{PE}, V^{\prime} / Q^{\prime}$ scan and serial ultrasonographic leg testing for DVT. Pulmonary angiography or lower limb venography were restricted to those patients in whom the noninvasive diagnostic protocol yielded inconclusive results. The overall prevalence of PE was 217 of 1,239 or $17 \%$ [25]. In the 665 patients in whom $\mathrm{PE}$ was excluded on the basis of a low/intermediate clinical probability, non-high-probability $V^{\prime} / Q^{\prime}$ scan and normal serial leg ultrasound test results, the 3-month thromboembolic risk was $0.5 \%$. This rate did not differ from that observed in patients with normal scan results $(0.6 \%)$ [25]. The strategy outlined, resulted in only 46 of 1,239 (4\%) patients requiring venography or pulmonary angiography for a definitive diagnosis [25].

In a companion paper [65], the Canadian investigators assessed the sensitivity and specificity of a rapid whole-blood D-dimer assay in 1,177 patients with suspected PE, and in subgroups of patients with low-clinical-probability or nondiagnostic $V^{\prime} / Q^{\prime}$ scans. In 703 patients who had been rated as having a low pretest probability, the likelihood ratio of a negative D-dimer test was 0.27 , resulting in a post-test probability of PE of 1\% [65]. Similarly, in 698 patients with nondiagnostic $V^{\prime} / Q^{\prime}$ scan results, the likelihood ratio of a negative $\mathrm{D}$-dimer test was 0.36 , resulting in a post-test probability of $2.8 \%$. None of the patients in whom PE was deemed absent on the basis of the noninvasive diagnostic protocol died of thromboembolic events during a 3-month follow-up [65]. It was concluded that anticoagulant therapy can be safely withheld in those patients with suspected PE in whom a negative D-dimer assay is associated with a low pretest probability or a nondiagnostic $V^{\prime} / Q^{\prime}$ scan [65].

The results of the aforementioned two studies should be interpreted with caution because: 1) the prevalence of PE was remarkably lower than that of other prospective studies [23, 24]; 2) the diagnostic yield of serial ultrasound studies was extremely low (conversion in 14 of 679 patients, or $2 \%$ ), thereby making this strategy unlikely to be cost-effective; and $3)$ the sensitivity of the whole-blood D-dimer assay $(85 \%)$ was lower than that of ELISA methods [64].

Recently, a prospective outcome study was carried out to validate a diagnostic protocol for suspected venous thromboembolism (DVT or PE) combining assessment of clinical probability, rapid D-dimer ELISA, single ultrasound venous study and $V^{\prime} / Q^{\prime}$ scan [82]. Venous thromboembolism was excluded by a negative D-dimer test in 286 of $918(31 \%)$ patients, whereas it was confirmed by lower limb ultrasonography in $157(17 \%)$. Venous thromboembolism was also deemed absent in patients with a low clinical probability of DVT and negative ultrasound result $(26 \%)$, and in patients with a low clinical probability of $\mathrm{PE}$ and nondiagnostic $V^{\prime} / Q^{\prime}$ scan results $(12 \%)$. The lung scan was diagnostic in 80 of the remaining patients (normal in 37, high probability in 43). Hence, a noninvasive diagnosis or exclusion of venous thromboembolism was possible in $866(94 \%)$ patients of the entire cohort [82]. In patients who did not receive anticoagulants, based on the results of the diagnostic protocol, the 3-month thromboembolic risk was $1.8 \%$ [82].

Even though the results of this study are impressive, it should be remembered that the proposed diagnostic strategy applies only to outpatients presenting in the accident and emergency department with suspected venous thromboembolism. In hospitalized patients, however, as demonstrated by the same group of investigators, both the D-dimer and the ultrasound test are much less effective [83]. Furthermore, in these studies [25, 65, 82], DVT was considered, for diagnostic purposes, equivalent to PE. This may have potentially harmful consequences in prognostic terms. As already mentioned, the results of a populationbased longitudinal study [10] indicate that: 1) the survival rate after venous thromboembolism (and particularly after PE) is significantly less than the expected survival of individuals of comparable age and sex; and 2) compared with DVT alone, PE, with or without DVT, after adjusting for comorbid conditions, is a significant and independent predictor of reduced survival until 3 months after diagnosis. Furthermore, the risk of early death (within 1 week) in patients with proven PE is $\sim 18$-fold higher than that of patients with DVT alone. Every effort should, therefore, be made to diagnose or exclude PE in patients in whom this entity is suspected. 
The PISA-PED study was designed to determine the sensitivity and specificity of the perfusion lung scan (without ventilation imaging) in patients with suspected PE and to develop a standardized diagnostic algorithm to assess the pretest clinical probability of $\mathrm{PE}$ prior to further objective testing [24, 26]. In 583 patients in whom pulmonary angiography was used as reference diagnostic standard, a high or intermediate clinical probability combined with a perfusion scan compatible with PE gave a 98\% positive predictive value [26]. Conversely, a low pretest probability paired with an abnormal scan not compatible with PE had a 99\% negative predictive value [26]. In those patients in whom clinical probability and lung scan findings were discordant (119 of 583 , or $20 \%$ ), the prevalence of PE ranged 10-71\% [26].

It appears, therefore, that a noninvasive strategy based on combining the standardized assessment of clinical probability with the perfusion lung scan would help restrict the need for angiography to a minority of patients suspected of having PE. By adopting this strategy, the proportion of patients requiring pulmonary angiography for a definitive diagnosis would undoubtedly be higher than that reported in the prospective studies quoted previously [25, 65, 82]. However, the following should be borne in mind. 1) The PISA-PED study was aimed at validating a noninvasive strategy for diagnosing $\mathrm{PE}$ and not venous thromboembolism. 2) The prevalence of $\mathrm{PE}$ in the PISA-PED study (39\%) was substantially higher than that of the other studies. 3) Most ( $>80 \%)$ patients were hospitalized at the time of study entry. 4) Nearly half of the patients had undergone recent surgery or trauma, nearly $25 \%$ had prior cardiovascular disease (including ischaemic heart disease and stroke) and some $20 \%$ had active malignancies. Thus, for reasons already given, the D-dimer test and lower limb ultrasonography would probably be much less effective than reported in outpatients $[25,65,82]$. 5) The predictive accuracy of both the $Q^{\prime}$ scan [24] and clinical evaluation [26] were prospectively assessed in comparison with conventional pulmonary arteriograms obtained at the same time.

\section{A noninvasive algorithm based on clinical probability and pulmonary vascular imaging techniques}

The application of a diagnostic strategy based on clinical evaluation and chest imaging techniques for the noninvasive diagnosis or exclusion of $\mathrm{PE}$ in the 750 consecutive patients enrolled in the PISA-PED trial [26] is summarized in figure 1. Confirmation or exclusion of PE and, hence, the predictive accuracy of the algorithm was based on the results of pulmonary angiography in the 583 patients with abnormal $Q^{\prime}$ scan results. PE was excluded on the basis of a normal or near normal $Q^{\prime}$ scan in the remaining 167 patients.

Pretest clinical probability of $\mathrm{PE}$ was assigned according to the standardized clinical model of table 2. $Q^{\prime}$ scan interpretation was performed according to the scintigraphic criteria reported in table 3. For the computation of the predictive accuracy of spiral computed tomographic angiography in the last

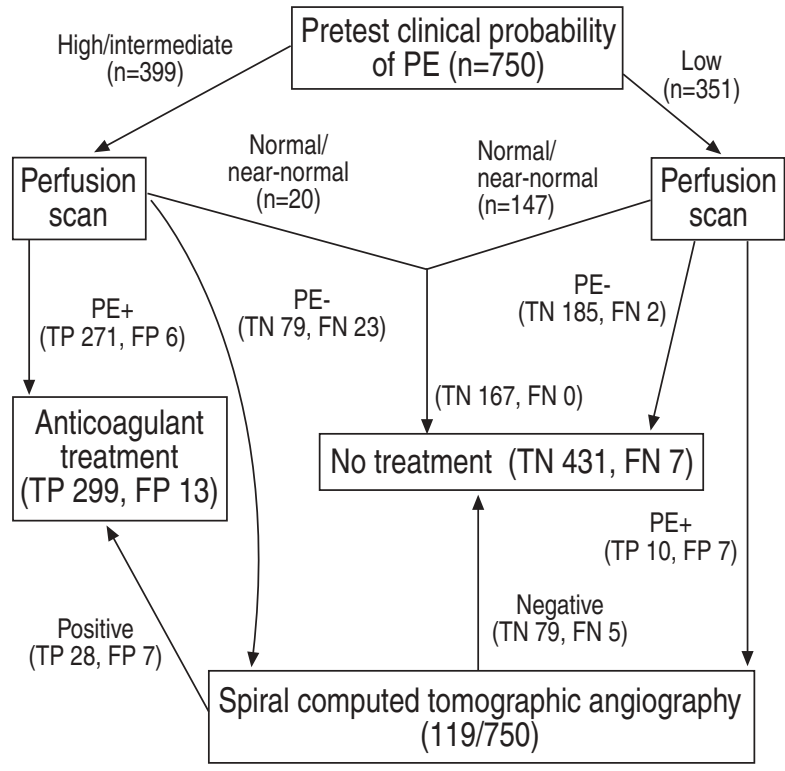

Fig. 1.- Noninvasive diagnostic algorithm based on clinical evaluation, perfusion scan and spiral computed tomographic angiography applied to 750 patients enrolled in the PISA-PED study [26]. Pretest clinical probability was assessed according to table 2 [26]. Perfusion scans were read according to the criteria reported in table 3 [24]. The predictive accuracy of the perfusion scan in terms of true positive (TP), false positive (FP), true negative (TN) and false negative (FN) was assessed by conventional pulmonary angiography $[24,26]$. The sensitivity $(85 \%)$ and specificity $(92 \%)$ of the spiral computed tomographic angiography were computed from the meta-analysis of RATHBUN et al. [59]. PE+: perfusion scan suggestive of pulmonary embolism (PE); PE-: perfusion scan not suggestive of $\mathrm{PE}$.

step of the diagnostic algorithm, a sensitivity of $85 \%$ and a specificity of $92 \%$ were assumed [59].

In the first step of the algorithm, the cohort of patients suspected of having PE could be almost equally divided into patients with a high or intermediate pretest clinical probability $(53 \%)$ and those with a low pretest probability $(47 \%)$ of the disease.

Normal/near-normal perfusion scan results were found in approximately a quarter (22\%) of the patients. In these patients, the diagnostic work-up can be terminated. The vast majority of patients with normal/near-normal $Q^{\prime}$ scan results $(88 \%)$ had a low pretest clinical probability. Most patients (94\%) with a $Q^{\prime}$ scan suggestive of $\mathrm{PE}$ were among those with a high or intermediate clinical probability. This association of clinical and $Q^{\prime}$ scan findings, found in $37 \%$ of patients, had a $98 \%$ positive predictive value for PE and allowed anticoagulant treatment to be started immediately. Conversely, the majority of patients $(65 \%)$ with a $Q^{\prime}$ scan not suggestive of $\mathrm{PE}$ were among those with a low clinical probability. This association of clinical and $Q^{\prime}$ scan findings, found in $25 \%$ of patients, had a $99 \%$ negative predictive value for PE and allowed anticoagulant treatment to be withheld. Thus, after pretest clinical evaluation and $Q^{\prime}$ scan, clinical decision making was complete in 631 of $750(84 \%)$ patients, those with normal/near-normal scan results and those with concordant clinical and $Q^{\prime}$ scan findings. 
Table 4. - Evidence-based criteria for the confirmation or exclusion of pulmonary embolism (PE)

\begin{tabular}{|c|c|}
\hline PE present & PE absent \\
\hline Positive pulmonary angiography & Negative pulmonary angiography \\
\hline $\begin{array}{l}\text { Positive computed tomographic } \\
\text { pulmonary angiography }\end{array}$ & Normal/near-normal perfusion scan \\
\hline $\begin{array}{l}\text { Perfusion scan suggestive of PE and } \\
\text { high/intermediate pretest clinical probability }\end{array}$ & $\begin{array}{l}\text { Perfusion scan not suggestive of PE and } \\
\text { low pretest clinical probability }\end{array}$ \\
\hline $\begin{array}{l}\text { Positive echocardiography and high/intermediate } \\
\text { pretest clinical probability }\end{array}$ & $\begin{array}{l}\text { D-dimer }<500 \mu \mathrm{g} \cdot \mathrm{L}^{-1} \text {, negative lower limb compression } \\
\text { ultrasonography and low pretest clinical probability }\end{array}$ \\
\hline
\end{tabular}

Among the remaining 119 (16\% of the cohort) patients with discordant clinical and $Q^{\prime}$ scan findings, the post-test probability of PE was neither sufficiently high nor sufficiently low to permit therapeutic decisions to be made without further testing. Most (86\%) of these patients had a high or intermediate pretest clinical probability and an abnormal $Q^{\prime}$ scan result not suggestive of PE. Among patients with a low pretest clinical probability, the diagnostic work-up was concluded after the perfusion scan in 334 of 351 $(95 \%)$. Only 12 of $351(3 \%)$ patients with low pretest clinical probability were found to have PE. Hence, a low pretest clinical probability has, of itself, a very high negative predictive value $(97 \%)$.

Execution of spiral computed tomographic angiography would be the last step of the proposed diagnostic algorithm in the 119 patients with discordant clinical and $Q^{\prime}$ scan findings. On angiography, $33(28 \%)$ of these patients were found to have PE. Assuming 84\% sensitivity and 92\% specificity, CT would have been positive for PE in 35 patients and negative in 84 . The positive and negative predictive values of this noninvasive diagnostic algorithm are 96 and $98 \%$, respectively. The overall predictive accuracy is $97 \%$. It should be borne in mind that it is assumed that all spiral computed tomographic angiographic examinations will yield conclusive results. In the presence of nondefinitive results, as may occur in $\sim 10 \%$ of cases, the diagnostic work-up should be terminated by pulmonary angiography. Conversely, with the expected increase in the predictive accuracy of spiral computed tomographic angiography with thinner slice collimation and multiple detection, it is conceivable that this diagnostic algorithm could possibly yield further improvements in performance.

Bearing in mind that an effective diagnostic strategy should be as flexible as possible in order to be applied in every clinical setting, a list of evidence-based criteria for the safe confirmation or exclusion of pulmonary embolism are proposed in table 4 . These criteria can be implemented in the diagnostic algorithm of figure 1 in relation to the experience of the attending physician, degree of severity of the patient's clinical condition, the availability of diagnostic equipment and specific logistics.

\section{References}

1. Morpurgo M, Schmid C. Clinico-pathological correlations in pulmonary embolism: a posteriori evaluation. Prog Respir Res 1980; 13: 8-15.
2. Karwinski B, Svendsen E. Comparison of clinical and postmortem diagnosis of pulmonary embolism. J Clin Pathol 1989; 42: 135-139.

3. Sperry KL, Key CR, Anderson RE. Toward a population-based assessment of death due to pulmonary embolism in New Mexico. Hum Pathol 1990; 21: 159-165.

4. Stein PD, Henry SW. Prevalence of acute pulmonary embolism among patients in a general hospital and at autopsy. Chest 1995; 108: 978-981.

5. Task Force Report of the European Society of Cardiology. Guidelines on diagnosis and management of acute pulmonary embolism. Eur Heart J 2000; 21: 1301-1336.

6. Silverstein MD, Heit JA, Mohr DN, Petterson TM, $\mathrm{O}^{\prime}$ Fallon WM, Melton LJ III. Trends in the incidence of deep vein thrombosis and pulmonary embolism. Arch Intern Med 1998; 158: 585-593.

7. Anderson FA, Wheeler HB, Goldberg $\mathrm{J}$, et al. A population-based perspective of the hospital incidence and case-fatality rates of deep vein thrombosis and pulmonary embolism. Arch Intern Med 1991; 151: 933-938.

8. Diamond GA, Forrester JS. Analysis of probability as an aid in the clinical diagnosis of coronary artery disease. New Engl J Med 1979; 300: 1350-1358.

9. Egermayer P. Follow-up for death or recurrence is not a reliable way of assessing the accuracy of diagnostic tests for thromboembolic disease. Chest 1997; 111: $1410-1413$.

10. Heit JA, Silverstein MD, Mohr DN, Petterson TM, $\mathrm{O}^{\prime}$ Fallon WM, Melton JM III. Predictors of survival after deep vein thrombosis and pulmonary embolism. A population-baed, cohort study. Arch Intern Med 1999; 159: 445-453.

11. Moser KM. Venous thromboembolism. Am Rev Respir Dis 1990; 141: 235-249.

12. Hyers TM. Venous thromboembolism. Am J Respir Crit Care Med 1999; 159: 1-14.

13. Bell WRT, Simon TL, DeMets DL. The clinical features of submassive and massive pulmonary emboli. Am J Med 1977; 62: 355-360.

14. Stein PD, Willis PW III, DeMets DL. History and physical examination in acute pulmonary embolism in patients without pre-existing cardiac or pulmonary disease. Am J Cardiol 1981; 47: 218-223.

15. Stein PD, Terrin ML, Hales CA, et al. Clinical, laboratory, roentgenographic, and electrocardiographic findings in patients with acute pulmonary embolism and no pre-existing cardiac or pulmonary disease. Chest 1991; 100: 598-603.

16. Stein PD, Saltzman HA, Weg JG. Clinical characteristics of patients with acute pulmonary embolism. Am J Cardiol 1991; 68: 1723-1724. 
17. Palla A, Donnamaria V, Petruzzelli S, Rossi G, Riccetti G, Giuntini C. Enlargement of the right descending pulmonary artery in pulmonary embolism. Am J Roentgenol 1983; 141: 513-517.

18. Westermark N. On the roentgen diagnosis of lung embolism. Acta Radiol 1938; 19: 357-372.

19. Hampton AO, Castleman B. Correlation of postmortem teleroentgenograms with autopsy finding: with special reference to pulmonary embolism and infarction. Am J Roentgenol 1940; 43: 305-326.

20. McGinn S, White PD. Acute cor pulmonale resulting from pulmonary embolism. JAMA 1935; 104: 14731480.

21. Stein PD, Dalen JE, McIntyre KM, Sasahara A, Wenger NK, Willis PW III. The electrocardiogram in acute pulmonary embolism. Progr Cardiovasc Dis 1975; 12: 247-257.

22. Santolicandro A, Prediletto R, Fornai E, et al. Mechanisms of hypoxemia and hypocapnia in pulmonary embolism. Am J Respir Crit Care Med 1995; 152: 336-347.

23. PIOPED Investigators. Value of the ventilationperfusion scan in acute pulmonary embolism: results of the Prospective Investigation of Pulmonary Embolism Diagnosis (PIOPED). JAMA 1990; 263: $2753-$ 2759.

24. Miniati M, Pistolesi M, Marini C, et al. Value of perfusion lung scan in the diagnosis of pulmonary embolism: results of the Prospective Investigative Study of Acute Pulmonary Embolism Diagnosis (PISA-PED). Am J Respir Crit Care Med 1996; 154: 1387-1393.

25. Wells PS, Ginsberg JS, Anderson DR, et al. Use of a clinical model for safe management of patients with suspected pulmonary embolism. Ann Intern Med 1998; 129: 995-1005.

26. Miniati M, Prediletto R, Formichi B, et al. Accuracy of clinical assessment in the diagnosis of pulmonary embolism. Am J Respir Crit Care Med 1999; 159: 864 871.

27. Wicki J, Perneger TV, Junod AF, Bounameaux H, Perrier A. Assessing clinical probability of pulmonary embolism in the emergency ward: a simple score. Arch Intern Med 2001; 161: 92-97.

28. Quinn DA, Thomson BT, Terrin ML, et al. A prospective investigation of pulmonary embolism in women and men. JAMA 1992; 268: 1689-1696.

29. Worsley DF, Alavi A, Aronchick JM, et al. Chest radiographic findings in patients with acute pulmonary embolism: observations from the PIOPED study. Radiology 1993; 189: 133-136.

30. Rodger MA, Carrier M, Jones GN, et al. Diagnostic value of arterial blood gas measurement in suspected pulmonary embolism. Am J Respir Crit Care Med 2000; 162: 2105-2108.

31. Remy-Jardin M, Remy J, Wattinne L, Giraud F. Central pulmonary thromboembolism: diagnosis with spiral volumetric CT with single breathhold technique. Comparison with pulmonary angiography. Radiology 1992; 185: 381-387.

32. Woodard PK. CT scan negative for pulmonary embolism: where do we go from here? Radiology 2000; 215: 325-326.

33. Bates SM, Ginsberg JS. Helical computed tomography and the diagnosis of pulmonary embolism. Ann Intern Med 2000; 132: 240-241.

34. Stein PD. Observations in venous thromboembolism. Am J Med 2001; 110: 69-70.
35. Royal HD, Siegel BA. Use of imaging for the diagnosis of pulmonary embolism. Radiology 2001; 218: 912-913.

36. Pattynama PT. Meaning of an helical CT angiogram negative for pulmonary embolism. Radiology 2001; 218: 913-916.

37. Meaney JFM, Weg JG, Chevenert TL, StaffordJohnson D, Hamilton BH, Prince MR. Diagnosis of pulmonary embolism with magnetic resonance angiography. New Engl J Med 1997; 336: 1422-1427.

38. Comroe JH Jr. The main functions of the pulmonary circulation. Circulation 1966; 33: 146-158.

39. Kipper MS, Moser KM, Kortman KE, Ashburn WL. Long term follow-up in patients with suspected pulmonary embolism and a normal lung scan. Chest 1982; 82: 411-415.

40. Hull RD, Raskob GE, Coates G, Panju AA. Clinical validity of a normal perfusion lung scan in patients with suspected pulmonary embolism. Chest 1990; 97: 23-26.

41. van Beek EJR, Kuyer PMM, Schenk BE, Brandjes DPM, ten Cate JW, Buller HR. A normal perfusion scan in patients with clinically suspected pulmonary embolism. Frequency and clinical validity. Chest 1995 ; 108: $170-173$.

42. Oudkerk M, van Beek EJR, van Putten WLJ, Rutten FFH. Management strategies in patients with suspected pulmonary embolism. In: Oudkerk $\mathrm{M}$, van Beek EJR, ten Cate JW, eds. Pulmonary Embolism. Berlin, Blackwell Science Ltd, 1999; pp. 410-429.

43. Kim KI, Muller NL, Mayo JR. Clinically suspected pulmonary embolism: utility of spiral CT. Radiology 1999; 210: 693-697.

44. Lipchik RJ, Goodman LR. Spiral computed tomography in the evaluation of pulmonary embolism. Clin Chest Med 1999; 20: 731-738.

45. Lomis NN, Yoon HC, Moran AG, Miller FJ. Clinical outcomes of patients after a negative spiral CT pulmonary arteriogram in the evaluation of acute pulmonary embolism. J Vasc Interv Radiol 1999; 10 : 707-712.

46. Garg K, Sieler H, Welsh CH, Johnston RJ, Russ PD. Clinical validity of helical CT being interpreted as negative for pulmonary embolism: implications for patient treatment. Am J Roentgenol 1999; 172: 1627 1631.

47. McEwan L, Gandhi M, Andersen J, Manthey K. Can CT pulmonary angiography replace ventilationperfusion scans as a first line investigation for pulmonary emboli? Australas Radiol 1999; 43: 311-314.

48. Lorut C, Ghossains M, Horellou MH, Achkar A, Fretault J, Laaban JP. A noninvasive diagnostic strategy including spiral computed tomography in patients with suspected pulmonary embolism. Am J Respir Crit Care Med 2000; 162: 1413-1418.

49. Qanadli SD, Hajjam ME, Mesurolle B, et al. Pulmonary embolism detection: prospective evaluation of dual-section helical CT versus selective pulmonary arteriography in 157 patients. Radiology 2000; 217: 447-455.

50. Blachere H, Latrabe V, Mountadon M, et al. Pulmonary embolism revealed on helical CT angiography: comparison with ventilation-perfusion radionuclide lung scanning. Am J Roentgenol 2000; 174: 1041-1047.

51. Goodman LR, Lipchik RJ, Kuzo RS, Liu Y, McAuliffe TL, O'Brien DJ. Subsequent pulmonary embolism: risk after a negative helical CT pulmonary 
angiogram. Prospective comparison with scintigraphy. Radiology 2000; 215: 535-542.

52. Baile EM, King GG, Muller NL, et al. Spiral computed tomography is comparable to angiography for the diagnosis of pulmonary embolism. Am J Respir Crit Care Med 2000; 161: 1010-1015.

53. Goodman PC. Spiral CT for pulmonary embolism. Semin Respir Crit Care Med 2000; 21: 503-510.

54. Ryu JH, Swensen SJ, Olson EJ, Pellikka PA. Diagnosis of pulmonary embolism with use of computed tomographic angiography. Mayo Clin Proc 2001; 76: 59-65.

55. Paterson DI, Schwartzam K. Strategies incorporating spiral CT for the diagnosis of pulmonary embolism. A cost-effectivenss analysis. Chest 2001; 119: 1791-1800.

56. Ost D, Rozenshtein A, Saffran L, Snider A. The negative predictive value of spiral computed tomography for the diagnosis of pulmonary embolism in patients with nondiagnostic ventilation-perfusion scans. Am J Med 2001; 110: 16-21.

57. Stein PD, Hull RD, Raskob GE. Withholding treatment in patients with acute pulmonary embolism who have a high risk of bleeding and negative serial noninvasive leg tests. Am J Med 2000; 109: 301-306.

58. Shah AA, Davis SD, Gamsu G, Intriere L. Parenchymal and pleural findings in patients with and patients without acute pulmonary embolism detected at spiral CT. Radiology 1999; 211: 147-153.

59. Rathbun SW, Raskob GE, Whitsett TL. Sensitivity and specificity of helical computed tomography in the diagnosis of pulmonary embolism: a systematic review. Ann Intern Med 2000; 132: 227-232.

60. Mullins MD, Becker DM, Hagspiel KD, Philbrick JT. The role of spiral volumetric computed tomography in the diagnosis of pulmonary embolism. Arch Intern Med 2000; 160: 293-298.

61. Harvey RT, Gefter WB, Hrung JM, Langlotz CP. Accuracy of CT angiography versus pulmonary angiography in the diagnosis of acute pulmonary embolism: evaluation of the literature with summary ROC curve analysis. Acad Radiol 2000; 7: 786-797.

62. van Beck EJR, Brouwers EM, Song B, Bongaerts AH, Oudkerk M. Lung scintigraphy and helical computed tomography for the diagnosis of pulmonary embolism: a meta-analysis. Clin Appl Thromb Hemost 2001; 7: 87-92.

63. Perrier A, Howarth N, Didier D, et al. Performance of helical computed tomography in unselected outpatients with suspected pulmonary embolism. Ann Intern Med 2001; 135: 88-97.

64. Bounameaux H, de Moerloose P, Perrier A, Reber G. Plasma measurement of $\mathrm{D}$-dimer as diagnostic aid in suspected venous thromboembolism: an overview. Thromb Haemost 1994; 71: 1-6.

65. Ginsberg JS, Wells PS, Kearon C, et al. Sensitivity and specificity of a rapid whole-blood assay for D-dimer in the diagnosis of pulmonary embolism. Ann Intern Med 1998; 129: 1006-1011.

66. Hein-Rasmussen R, Tuxen CD, Wiinberg N. Diagnostic value of the Nycocard, Nycomed D-dimer assay for the diagnosis of deep vein thrombosis and pulmonary embolism: a retrospective study. Thromb Res 2000; 100: 287-292.

67. Duriex P, Dhote R, Meyniard O, Spaulding C, Luchon L, Toulon P. D-dimer testing as the initial test for suspected pulmonary embolism. Appropriateness of prescription and physician compliance to guidelines. Thromb Res 2001; 101: 261-266.

68. Lensing AWA, Prandoni P, Brandjes D, et al. Detection of deep vein thrombosis by real-time B-mode ultrasonography. N Engl J Med 1989; 320: 342-345.

69. Kearon C, Ginsberg JS, Hirsh J. The role of venous ultrsonography in the diagnosis of suspected deep vein thrombosis and pulmonary embolism. Ann Intern Med 1998; 129: 1044-1049.

70. Hull RD, Hirsh J, Carter CJ, et al. Pulmonary angiography, ventilation lung scanning, and venography for clinically suspected pulmonary embolism with abnormal perfusion scan. Ann Intern Med 1983; 98: 891-899.

71. Meyerovitz MF, Mannting F, Polak JF, Goldhaber SZ. Frequency of pulmonary embolism in patients with low-probability lung scan and negative lower extremity venous ultrasound. Chest 1999; 115: 980982.

72. Daniel KR, Jackson RE, Kline JA. Utility of lower extremity venous ultrasound scanning in the diagnosis and exclusion of pulmonary embolism in outpatients. Ann Emerg Med 2000; 35: 547-554.

73. Goldhaber SZ. Pulmonary embolism. New Engl J Med 1998; 339: 93-104.

74. Goldhaber SZ, Visani L, De Rosa M. Acute pulmonary embolism: clinical outcomes in the International Cooperative Pulmonary Embolism Registry (ICOPER). Lancet 1999; 353: 1386-1389.

75. Nazeyrollas P, Metz D, Jolly D, et al. Use of thransthoracic Doppler echocardiography combined with clinical and electrocardiographic data to predict acute pulmonary embolism. Eur Heart $J$ 1996; 17: 779-786.

76. Perrier A, Tamm C, Unger PF, et al. Diagnostic accuracy of Doppler-echocardiography in unselected patients with suspected pulmonary embolism. Int J Cardiol 1998; 65: 101-109.

77. Grifoni S, Olivotto I, Cecchini P, et al. Utility of an integrated clinical, echocardiographic, and venous ultrasonographic approach for triage of patients with suspected pulmonary embolism. Am J Cardiol 1998; 82: $1230-1235$.

78. Miniati M, Monti S, Pratali L, et al. Value of transthoracic echocardiography in the diagnosis of pulmonary embolism: results of a prospective study in unselected patients. Am J Med 2001; 110: 528-535.

79. Kasper W, Konstantinides S, Geibel A, Tiede N, Krause T, Just H. Prognostic significance of right ventricular afterload stress detected by echocardiography in patients with clinically suspected pulmonary embolism. Heart 1997; 77: 346-349.

80. Ribeiro A, Lindmarker P, Juhlin-Dannfelt A, et al. Echocardiography Doppler in pulmonary embolism: right ventricular dysfunction as a predictor of mortality rate. Am Heart J 1997; 134: 479-487.

81. Ribeiro A, Lindmarker P, Johnsson H, et al. Pulmonary embolism. One-year follow-up with echocardiography Doppler and five-year survival analysis. Circulation 1999; 99: 1325-1330.

82. Perrier A, Desmarais S, Miron MJ, et al. Non-invasive diagnosis of venous thromboembolism in outpatients. Lancet 1999; 353: 190-195.

83. Miron MJ, Perrier A, Bounameaux H, et al. Contribution of noninvasive evaluation to the diagnosis of pulmonary embolism in hospitalized patients. Eur Respir J 1999; 13: 1365-1370. 